\title{
Placement à des fins d'assistance en établissement psychiatrique
}

Helena Hermann, Dr sc. méd.; Paul Hoff, Prof.-Dr phil.; Florian Hotzy, Dr méd.; Lena Schneller, lic. iur.; Anastasia Theodoridou, Dr méd.; Matthias Jäger, PD Dr méd.

Clinique psychiatrique universitaire de Zurich, centre de compétence pour le droit de la protection de l'enfant et de l'adulte

Pour faciliter la lecture du document, le masculin générique est utilisé pour désigner les deux sexes.

Les résultats détaillés du sondage sont en ligne sur: www.dppp.uzh.ch/en/ news/fuersorgerische unterbringung.html
Certaines crises psychiques peuvent mettre une personne dans un état exceptionnel qui rend nécessaire un placement forcé dans un établissement adéquat, afin de la protéger. Ce type de placement à des fins d'assistance (PLAFA) est une importante entrave au droit à l'autodétermination de la personne concernée. Conformément à l'art. 426 CC, il ne doit donc être ordonné qu'en dernier ressort, si le traitement visant à mettre la personne hors de danger ne peut être assuré par d'autres mesures moins radicales, comme un traitement ou un suivi en ambulatoire.

Etant donné la portée d'un PLAFA sur la liberté de la personne concernée, le taux de PLAFA doit rester le plus faible possible, notamment au regard de la promotion de l'autonomie du patient. L'harmonisation des législations cantonales est également importante pour garantir un traitement identique de toutes les personnes concernées. C'est dans cette optique d'harmonisation que l'actuel droit de la protection de l'enfant et de l'adulte est entré en vigueur au début de l'année 2013. Malgré cette révision de la loi, les différences de méthodes sont encore parfois considé-

\section{Résumé}

Un placement à des fins d'assistance (PLAFA) est une entrave importante au droit à l'autodétermination de la personne concernée. Des efforts d'harmonisation des principes juridiques sont faits, notamment avec le droit actuel de la protection de l'enfant et de l'adulte, entré en vigueur en 2013. Pourtant, étant donné les spécificités des législations et structures cantonales, on constate encore de considérables différences entre les cantons et entre les établissements eux-mêmes dans la gestion du PLAFA. Un sondage auprès des médecins-chefs des institutions psychiatriques de Suisse a permis de synthétiser les difficultés et les différences de la mise en pratique. Les pertes de qualité dues au manque de standards de formation posent des problèmes non négligeables, mais aussi l'absence de disponibilité et de disposition des médecins à ordonner un PLAFA et l'indépendance de I'instance décisionnelle. A cela s'ajoute I'hétérogénéité des procédures dans les situations exceptionnelles, en cas de statut juridique flou de la personne placée, ainsi que le risque d'instrumentalisation de la psychiatrie qu'entraînent certaines attentes de la société. rables entre les cantons et d'un établissement à l'autre. S'ajoutent à cela, concernant le placement à des fins d'assistance, des difficultés liées à la mise en pratique des dispositions légales, les lacunes de la réglementation, ainsi que les aspects juridiques, éthiques et sociétaux, dont il convient de débattre de façon critique. Les problématiques suivantes sont examinées, en partant des échanges avec les membres de l'Association suisse des médecins-cheffes et médecins-chefs en psychiatrie (ASMP), et du sondage mené auprès d'eux. ${ }^{1}$

\section{Pertes de qualité dues au manque de standards de formation}

Les compétences permettant d'ordonner un PLAFA sont régies par le droit de la protection de l'enfance et de l'adulte de chacun des cantons. Outre les autorités de protection de l'enfant et de l'adulte (APEA), certains médecins sont aussi habilités à ordonner un PLAFA. Selon la législation, la qualification et l'expérience requises font plus ou moins défaut à l'instance décisionnelle. Dans certains cantons, tous les médecins autorisés à pratiquer à titre indépendant, quelle que soit leur spécialité, ont le droit d'ordonner un internement par PLAFA; dans d'autres cantons, seuls certains spécialistes, urgentistes ou médecins officiels sont habilités à le faire. Il est logique que les différences de qualifications et d'expérience entraînent des décisions de qualité variable; mais on constate régulièrement des pertes de qualité dues soit au manque d'expertise psychiatrique, soit au manque de connaissances du contexte juridique (voir [1, 2]). Une gestion plus restrictive des compétences ou une obligation de certification pourrait harmoniser la procédure; cela exigerait de définir un standard minimum de connaissances et de compétences pour pouvoir ordonner un PLAFA.

\section{Absence de disponibilité et de disposition des médecins à prescrire un PLAFA}

D’après l'art. $427 \mathrm{CC}$, les personnes admises de leur plein gré peuvent être retenues dans l'établissement 
maximum 72 heures contre leur volonté, en cas de mise en danger d'elles-mêmes ou d'autrui. Pour prolonger l'hospitalisation contre la volonté de la personne, il faut qu'une décision exécutoire soit prononcée dans ce délai. La sollicitation d'un spécialiste pour ordonner un PLAFA une fois que la personne est retenue pose un grave problème dans certains endroits: étant donné le manque de disponibilité ou de disposition des spécialistes à ordonner le placement, parfois aucune décision de placement n'est prise avant expiration du délai. Le personnel soignant est alors confronté à un lourd dilemme éthique, au cours duquel le devoir d'assistance entre en conflit avec le devoir de garantie de la liberté de la personne concernée. La mise en pratique des dispositions législatives liées au maintien des personnes dans l'établissement n'est donc pas facile partout. Pour résoudre ce problème, il est nécessaire que des spécialistes qualifiés soient disponibles dans les délais légaux.

\section{Atteinte à l'indépendance de l'instance décisionnelle}

Au problème de disponibilité s'ajoute la question de l'indépendance de l'instance qui ordonne par rapport à celle qui accueille, notamment concernant les décisions de PLAFA après retenue de la personne. Quand il est difficile de faire intervenir des médecins extérieurs, et que les autorités ne sont pas disponibles, la nécessité amène parfois à recourir aux spécialistes de l'établissement lui-même ou à ceux d'un autre service. Ces difficultés concernent notamment les régions isolées, comme le canton GR, dans lesquelles on peut s'attendre à ce que le spécialiste extérieur sollicité ait un retard important, donc à ce que le traitement approprié soit rendu plus compliqué, voire impossible. L'atteinte à l'indépendance est aussi un problème dans les institutions relativement grandes, disposant de différents sites et établissements, et de services externes. Souvent, les personnes impliquées n'ont pas conscience qu'il s'agit d'une orientation dans leur propre institution. Se pose alors la question des critères qui déterminent l'indépendance.

Etant donné le manque de disponibilité de personnel qualifié pour décider un PLAFA, parfois des accords sont passés entre les autorités et les établissements, stipulant qu'en cas de blocage, les PLAFA peuvent être ordonnés par des médecins de l'établissement, à condition que ces derniers ne soient pas directement impliqués dans le traitement hospitalier. Ces stratagèmes posent des problèmes aux établissements et représentent une lourde charge pour les médecins des institutions. Il y a de grands risques que les médecins qui prennent la décision soient en difficulté. Notamment pour les décisions liées aux valeurs de la personne et quand le temps est compté, ainsi que quand les informations sont limitées, la mise en cause de l'évaluation d'un collègue n'est pas facile; elle l'est encore moins quand il s'agit d'une personne de la même institution. Mais il ne s'agit pas seulement de limiter le stress du corps médical. Il est aussi important de protéger le droit de la personne concernée à bénéficier d'un jugement par une deuxième instance indépendante, tout en notant que la garantie d'indépendance de l'instance décisionnelle et la garantie d'un traitement approprié peuvent entrer en conflit, et que dans ce cas, il sera nécessaire d'examiner attentivement le dossier.

\section{Prise en charge d'une mission régalienne par le corps médical}

Il faut en outre souligner que le spécialiste décidant d'un PLAFA assume une mission régalienne: le médecin restreint la liberté d'une personne. Cela peut entrer en conflit avec l'alliance thérapeutique, donc avoir une incidence défavorable sur le succès du traitement. Il faudrait examiner dans quelle mesure le corps médical doit et souhaite être le plus compétent pour assumer cette mission. Bien que, d'après le CC, le PLAFA soit une mesure administrative, actuellement, dans la plupart des cas, l'APEA n'est pas impliquée, ou relativement tardivement, au plus tard au bout de 6 semaines, lors du contrôle administratif du PLAFA. Si l'on compare avec les autres pays, cette durée est longue. Un contrôle rapide de la légitimité d'un PLAFA, avec une expertise psychiatrique, permettrait de soulager la double mission du corps médical, d'alléger la relation thérapeutique et pourrait contribuer à promouvoir l'autonomie des patients.

\section{Procédures hétérogènes dans les cas de statut juridique flou}

Il est aussi nécessaire de clarifier les situations exceptionnelles, dans lesquelles le statut juridique des personnes placées n'est pas clair lors de l'admission. Il n'est pas rare que les personnes déclarées comme placées à des fins d'assistance arrivent dans l'établissement sans décision écrite de PLAFA. Suivant les établissements, du point de vue formel, soit ces personnes sont immédiatement prises en charge comme des cas de PLAFA normaux, soit elles ne sont pas admises mais sont dans un premier temps retenues jusqu'à l'obtention d'une décision de PLAFA écrite. Il est aussi relativement fréquent que des personnes qui n'opposent aucune résistance, mais dont la capacité de jugement est altérée, soient placées dans un établissement sans qu'il 
y ait de décision écrite de PLAFA. Dans ces cas, la procédure est très variable. On peut citer entre autres des décisions de placement avant admission, des admissions régulières de patients de leur plein gré, ou des signatures de bons d'admission de plein gré par les proches. Parfois les procédures ne sont donc pas prévues dans la législation. Il serait important d'harmoniser ces façons de faire, en les appuyant sur des principes juridiques clairs. Il faut noter qu'un traitement dans le cadre d'un PLAFA présente l'avantage d'une plus grande transparence, car une décision de PLAFA peut faire l'objet d'un recours et implique la possibilité de solliciter une instance d'évaluation indépendante.

\section{Le comptage dans les statistiques}

On connaît les limites de comparaison des statistiques de PLAFA, liées au manque d'exhaustivité et aux biais (voir [3, 4]). Outre les harmonisations citées, l'amélioration de la qualité des données appelle d'autres ajustements, par exemple en ce qui concerne les cas de PLAFA qui sont libérés dans les 24 heures. Ces cas ne sont pas toujours intégrés dans les données complémentaires sur la psychiatrie de l'Office fédéral de la statistique. De plus, pour une saisie exhaustive des données, il serait important de procéder à un enregistrement explicite et séparé des placements au cours des retenues et de ceux après retenue.

\section{Position de la société et instrumentali- sation de la psychiatrie}

Mais avant tout, dans le placement à des fins d'assistance, il s'agit de questions de positionnement et d'attentes sociétales par rapport à la psychiatrie, auxquelles se voient directement confrontés les spécialistes qui prennent les décisions quand ils examinent la personne concernée. La tendance problématique à "psychiatriser» les problèmes sociétaux, qui va jusqu'à instrumentaliser la psychiatrie, est de plus en plus claire. On assiste parfois au placement de personnes pour qui l'établissement psychiatrique n'est pas l'institution appropriée, car il s'agit essentiellement de problèmes sociaux ou somatiques, qui pourraient être résolus bien plus facilement dans un autre cadre. Dans ces cas-là, le placement se fait cependant avant tout en psychiatrie, considérée comme la principale discipline pour gérer les admissions sans consentement. Les aspects économiques peuvent aussi jouer un rôle central à cet égard. Il est nécessaire d'avoir un discours social qui s'inscrive dans la durée, afin de développer une position commune des acteurs politiques et médicaux, ainsi que des représentants des personnes déjà confrontées à un PLAFA, et de déterminer clairement quels sont les besoins correspondants.

Auteurs supplémentaires: Patrik Benz-Gartenmann, Dr méd. Lorenza Bolzani, Dr méd.; Benjamin Dubno, Dr méd.; Martin Hatzinger, Prof.; Urs Hepp, Prof.; Uwe Herwig, Prof.; Patrik Hochstrasser, Dr méd.; Wolfram Kawohl, Prof.; Georges Klein, Dr méd.; Tim Klose, Dr méd.; Julius Kurmann, Dr méd.; Robert Maier, Dr méd.; Thomas Maier, PD Dr méd.; Beat Nick, Dr méd.; Thomas Reisch, Prof.; Florian Riese, Dr méd.; Axel Ropohl, Dr méd.; Egemen Savaskan, Prof.; Andres Schneeberger, Dr méd.; Erich Seifritz, Prof.; RafaelTraber, Dr méd.

\section{Remerciements}

Nous remercions l'Association suisse des médecins-cheffes et médecins-chefs en psychiatrie (ASMP) pour sa coopération, ainsi que pour la présentation des résultats et les discussions à ce sujet lors de son congrès annuel, le 24 mars 2017, à la Klinik Zugersee.

\section{En l'absence de tout conflit d'intérêts.}

\section{Littérature}

1 Jäger M, Ospelt I, Kawohl W, Theodoridou A, Rössler W, Hoff P. Qualität unfreiwilliger Klinikeinweisungen in der Schweiz. Praxis. 2014;103: 631-9.

2 Kieber-Ospelt I, Theodoridou A, Hoff P, Kawohl W, Seifritz E, Jäger M. Quality Criteria for Involuntary Psychiatric Admissions before and after the introduction to the Law Governing Legal Protection of Minors and Adults (KESR). BMC Psychiatry. 2016;16: 291. DOI 10.1186/s12888-016-0998-z.

3 Schuler D, Tuch A, Buscher N, Camenzind P. Psychische Gesundheit in der Schweiz. Monitoring 2016 (Obsan Bericht 72). Neuchâtel: Schweizerisches Gesundheitsobservatorium; 2016.

4 Gassmann J. Wirksamkeit des Rechtsschutzes bei psychiatrischen Zwangseinweisungen in der Schweiz. Erstellt im Auftrag des Bundesamtes für Gesundheit. Bern: Bundesamt für Gesundheit; 2011 